\title{
Evaluation of methods to detect in vitro biofilm formation by staphylococcal clinical isolates
}

Sarita Manandhar ${ }^{1,2}$, Anjana Singh ${ }^{3}$, Ajit Varma2 ${ }^{2}$ Shanti Pandey ${ }^{4}$ and Neeraj Shrivastava $2,5^{*}$

\begin{abstract}
Objective: Staphylococcus genus comprising both Staphylococcus aureus and coagulase negative staphylococci (CONS) are widely distributed in nature and can infect diversity of hosts. Indeed, staphylococci are the major pathogens causing biofilm associated infections caused by contaminated hospital indwelling devices. These infections are persistent in nature being highly refractory to various stresses including antibiotics. Implementation of efficient diagnostic techniques for the biofilm production would help minimize the disease burden. Thus, early detection of pathogenic strains producing biofilms warrant the utmost importance in diagnostic laboratories especially in resource limited settings.

Result: Among 375 isolates collected from different clinical specimens, 214 (57\%) were identified as coagulase negative staphylococci and 161 (43\%) S. aureus. Detection of In-vitro biofilm formation in these isolates were carried out by three commonly used phenotypic assays and a genotypic assay. While evaluating the results, tissue-culture method with supplemented glucose and sucrose showed the best correlation with the results of genotypic assay.
\end{abstract}

Keywords: Staphylococcus spp., Biofilm, Clinical specimens, Phenotypic assays, Genotypic assay

\section{Introduction}

Staphylococcus spp., widely distributed in nature, colonize the skin and anterior nares of humans. However, upon achieving the favorable environment, they can infect the diversified hosts $[1,2]$ due to the presence of numerous virulence factors including exotoxins, enzymes, surface proteins, ability of biofilm production and acquisition of resistance to multiple drugs [3-5].

Biofilm is a structured community of bacterial cells enclosed in self-produced polymeric matrix adherent to an inert or living surface [6-8]. As implant devices are increasingly used in medical practice, staphylococcal infections are now considered one of the major nosocomial infections $[9,10]$. Biofilm associated infections are characteristically refractory to different stresses including

\footnotetext{
*Correspondence: nsrivastava1@amity.edu; neersar@gmail.com

${ }^{2}$ Amity Institute of Microbial Technology, Amity University Uttar Pradesh Noida, UP 201303, India

Full list of author information is available at the end of the article
}

host immune defense and antibiotics, leading to persistent infections [2, 11-13].

The polysaccharide intercellular adhesin (PIA) is the main biomolecule responsible for cell aggregation and biofilm formation. PIA biosynthesis is carried out by the proteins encoded by the ica operon (icaADBC) [14-16]. Given that staphylococcal infections associated with medical devices have significant impact on morbidity, mortality and socio-economic burden, prevention and management of such infections remains a priority. Thus, detection and differentiation of staphylococci in their ability to form biofilm in routine laboratory practice bear great importance to initiate effective treatment measures and minimize unsuccessful antibiotic therapies [7, 17] .

Various phenotypic methods like Congo-red agar method (CRA), tube method (TM), tissue culture plate method (TCP), electron microscopy, confocal scanning microscopy and bioluminescent assay are available for the detection of biofilm formation in staphylococcal infections $[7,18]$. Detection of biofilm related genes using PCR techniques have been increasingly used, but 
this may be infeasible as routine diagnostic in a resourcelimited country like Nepal. Therefore, in the present study, we sought to compare and evaluate the sensitivity and specificity of three most commonly used phenotypic assays with the genotypic assay to detect biofilm production.

\section{Main text}

\section{Materials and methods}

A total of 375 clinical staphylococcal isolates were collected from two tertiary care hospitals from 2015 to 2017. Staphylococci were isolated and identified from various clinical samples by standard microbiological techniques [19]. High biofilm producer strain Staphylococcus epidermidis ATCC 35984 was used as reference strain in all the tests performed. All experiments were performed in triplicate and repeated thrice.

\section{Screening of biofilm production Phenotypic assay}

The in vitro biofilm production was measured using phenotypic assays CRA, TM and TCP methods. In CRA method, biofilm production was measured qualitatively described [20]. The black colonies with dark consistency were regarded as strong biofilm producers while the pink colonies as biofilm non-producers.

TM, a qualitative method for the detection of biofilm formation was performed as described [21]. Briefly, biofilm formation was considered positive when a visible film was observed along the inner wall and bottom of tube. Depending on this, isolates were scored as $0,1,2$ and 3 for absence, weak, moderate and strong biofilm formation respectively.

TCP, a quantitative method was used as described by Christensen et al. with slight modification [21], using trypticase soy broth (TSB), TSB with $1 \%$ glucose and Brain Heart Infusion (BHI) broth with $2 \%$ sucrose. Optical densities (OD) of both the dry plates and eluted stain was measured using micro ELISA auto reader at OD $630 \mathrm{~nm}$. Mean OD value $<0.120,0.120-0.240$ and $>0.240$ were classified as non/weak, moderate and strong biofilm adherence respectively [18].

\section{Genotypic assay}

Polymerase chain reaction (PCR) was used to detect $i c a A$ and $i c a D$ genes. The genomic DNA was extracted using a DNA extraction kit following the manufacturer instructions (Thermo Fischer). The forward and reverse primers (Solis Biodyne, Denmark) for icaA used were 5'-TCTCTTGCAGGAGCAATCAA and 5'-TCAGGC ACTAACATCCAGCA respectively. For icaD, 5'-ATG GTCAAGCCCAGACAGAG as forward and $5^{\prime}$-CGT GTTTTCAACATTTAATGCAA as reverse primer. The
PCR product was analyzed in $2 \%$ agarose gel stained with SYBR safe (Invitrogen) dye [22].

\section{Statistical analysis}

Sensitivity and specificity were evaluated by comparing the result of phenotypic methods with genotypic methods as standard. Different phenotypic methods were also compared with TCP as standard for phenotypic assays. Chi square test was used to evaluate the apparent differences for significance at 95\% confidence level using IBM SPSS v 21.0.

\section{Results}

Based on coagulase test, we differentiated 375 isolates into 214 (57\%) CoNS and 161 (43\%) S. aureus. Among six CoNS species identified, S. epidermidis was the most prevalent (57.5\%), followed by S. saprophyticus (18.7\%), $S$. haemolyticus (11.2\%), S. hominis (7.0\%), S. capitis (5.6\%) (Additional file 1: Table S1).

Among 375 isolates, 86 (22.9\%) isolates were found to possess both icaA and icaD genes comprising 45 (28\%) S. aureus and 41 (19.2\%) CoNS which predominantly constituted 29 (33.7\%) S. epidermidis isolates (Table 1).

Among all isolates, 20 (5.3\%) isolates were positive in CRA while 329 (87.7\%) isolates were biofilm non-producers with red colonies. It was found that detection of biofilm production by TM method was statistically significant when compared with presence of ica genes whereas CRA and TCP methods were statistically insignificant (Table 1) (Additional file 2: Table S2). We observed $14 \%$ sensitivity and $88 \%$ specificity while comparing CRA method with the genotypic assay. This shows no good correlation of CRA method with genotypic assay (Table 3).

In TM method, $63(16.8 \%)$ isolates were found to be strong, 66 (17.6\%) moderate, and 246 (65.6\%) biofilm non-producers. The strong biofilm producers included 19 (11.8\%) S. aureus and 44 (20.6\%) CoNS species with highest frequency in S. epidermidis 33 (25.6\%). The sensitivity

\begin{tabular}{lllll}
$\begin{array}{l}\text { Table 1 Screening of } \\
\text { with different methods }\end{array}$ & in & vitro & biofilm & production \\
\hline $\begin{array}{l}\text { Biofilm } \\
\text { production }\end{array}$ & CRA & TM & TCP & Ica genes \\
\hline High & $20(5.3 \%)$ & $63(16.8 \%)$ & $21(5.6 \%)$ & $86(22.9 \%)$ \\
Moderate & $26(6.9 \%)$ & $66(17.6 \%)$ & $91(24.3 \%)$ & - \\
Weak/non & $329(87.7 \%)$ & $246(65.6 \%)$ & $263(70.1 \%)$ & $289(77.1 \%)$ \\
Pvalue & 0.390 & 0.000 & 0.374 & \\
\hline
\end{tabular}

CRA Congo Red Agar Method, TM Tube Method, TCP Tissue Culture Plate Method 
and specificity of the tube method showed $64 \%$ and $74 \%$ respectively to genotypic assay (Tables 1,3 ).

The TCP method was used to assess biofilm production using three variations in media. In TCP with TSB only, 21 (5.6\%) isolates with 4 (2.5\%) S. aureus and 17 (7.9\%) CoNS showed strong biofilm production. An addition of $1 \%$ glucose to TSB medium increased biofilm detection in 83 (22.1\%) comprising 48 (19.8\%) S. aureus and 35 (16.4\%) CoNS species. In BHI, incorporated with $2 \%$ sucrose also increased biofilm detection including 41 (25.5\%) $S$. aureus and 66 (30.8\%) CoNS species. Our study showed the induction of biofilm production on addition of nutrients specially glucose and sucrose. When TCP was compared with the genotypic assay, among 83 strong biofilm producers, $20(24.1 \%)$ were shown to possess icaAD genes. Our result showed no significant difference in biofilm production between dry plate and ethanol-eluted TCP method (Tables 2, 3; Additional file 3: Table S3).

The biofilm production is accurately confirmed by detecting the genes involved in biofilm formation. But PCR technique as routine diagnosis is impractical in resource-limited countries like Nepal. In this scenario, implementation of easier and reliable phenotypic method would be more appropriate. Therefore, we sought to evaluate CRA, TM, and modified TCP method with standard TCP method. The results revealed the CRA method with the highest specificity $(86 \%)$ but the lowest sensitivity (8\%). With that, the modified TCP method using BHI with $2 \%$ sucrose was $80 \%$ sensitive with $57 \%$ accuracy rate for differentiating biofilm producers and nonproducers. Addition of glucose in TSB corresponded to sensitivity and specificity of $59 \%$ when compared with the TCP method. These results suggested that modified TCP method using BHI with $2 \%$ sucrose and/or TSB supplemented with glucose, to be more reliable than those without supplements for detecting staphylococcal biofilm production (Additional file 4: Table S4).

\section{Discussion}

For high disease burden of biofilm associated staphylococcal infections, a reliable and prompt diagnostic method is essential in health care facilities $[2,23]$. Therefore, in this study, we evaluated three phenotypic, and a genotypic method of in vitro biofilm detection. To the best of our knowledge, this is first study using genotypic assay to detect in vitro biofilm production in clinical samples in Nepal.

In this study, 375 clinical staphylococcal isolates retained from various specimens were identified as $S$. aureus and CoNS in 161 (43\%) and 214 (57\%) isolates respectively. Consistent with previous studies, [24], $S$. epidermidis was the predominant CoNS species corresponding to $123(57.5 \%)$ isolates. Because of its adaptive ability and highest dominance on human skin and mucosa [25], S. epidermidis has been reported the most prevalent in multiple studies [26, 27].

A plethora of studies demonstrate the causal link between staphylococcal biofilm and the presence of ica operon [3, 28-33], which in turn are involved in the PIA production; the most extensively characterized staphylococcal biofilm component [7, 29, 34-36]. In the present study, concomitant presence of $i c a A$ and icaD genes was detected in $86(22.9 \%)$ staphylococcal isolates. Among CoNS, 29 (34\%) S. epidermidis isolates

Table 2 Frequency of biofilm production in TCP method with different media composition

\begin{tabular}{|c|c|c|c|c|c|c|}
\hline \multirow[t]{2}{*}{ Biofilm formation } & \multicolumn{2}{|l|}{ TSB only } & \multicolumn{2}{|c|}{$\mathrm{TSB}+$ glucose } & \multicolumn{2}{|c|}{$\mathrm{BHI}+$ sucrose } \\
\hline & Dry & Elution with ethanol & Dry & Elution with ethanol & Dry & Elution with ethanol \\
\hline High & $21(5.6 \%)$ & $22(5.9 \%)$ & $83(22.1 \%)$ & $87(23.2 \%)$ & $107(28.5 \%)$ & $97(25.9 \%)$ \\
\hline Moderate & $91(24.3 \%)$ & $85(22.7 \%)$ & $91(24.3 \%)$ & 109 (29.1\%) & $122(32.5 \%)$ & $132(35.2 \%)$ \\
\hline Weak/non & $263(70.1 \%)$ & 268 (71.5\%) & 201 (53.6\%) & 179 (47.7\%) & 146 (38.9\%) & 146 (38.9\%) \\
\hline
\end{tabular}

TSB, Tryptic Soy Broth; BHI, Brain Heart Infusion

Table 3 Statistical evaluation of phenotypic methods compared with genotypic method

\begin{tabular}{llllc}
\hline Screening methods & Sensitivity & Specificity & \multicolumn{2}{c}{$\begin{array}{l}\text { Positive predictive } \\
\text { value } \\
\text { value }\end{array}$} \\
\hline CRA & 14 & 88.2 & 26.1 & 77.5 \\
TM & 64 & 74.4 & 42.6 & 87.8 \\
TCP-dry & 33.7 & 71.3 & 26 & 72 \\
TCP-elution & 30.2 & 72 & 24.3 & 78.3 \\
\hline
\end{tabular}


found to possess icaAD genes. Los et al. showed the prevalence of ica operon in $27.4 \%$ nasopharyngeal $S$. epidermidis isolates from hospitalized patients [37]. Oliviera et al. detected ica genes in 40\% CoNS isolated from clinical specimen and nares of healthy individuals [7]. Likewise, Cafiso et al., Nasr et al. and deSilva et al. showed $37 \%, 32 \%$ and $40 \%$ staphylococcal isolates positive for $i c a$ genes respectively $[31,34,38]$.

CRA method showed slime production in 46 (12.2\%) staphylococcal isolates. The sensitivity and specificity of CRA method was only $14 \%$ and $88 \%$ respectively as compared to genotypic assay. Arciola and colleagues also identified eight and six CRA negative isolates possessing ica genes in two consecutive studies [16, 39]. Similarly, Cafiso et al. and Fitzpatrick et al. also showed the reduced accuracy of this method to biofilm production $[34,40]$. All these evidences suggest that, despite being easier and faster, CRA method cannot be relied upon for precise detection of biofilm producers in routine diagnostic laboratory.

TM showed $63(16.8 \%)$ isolates as strong, 66 (17.6\%) moderate and 246 (65.6\%) weak/non-biofilm producers. The TM results showed $64 \%$ sensitivity and $74 \%$ specificity as compared to the genotypic assay. Consistence with the previous study [7], TM among phenotypic assays in our study demonstrated the best correlation with genotype assay.

The expression of ica genes in vitro studies have been reported to be highly variable depending on the composition of media as their expression is induced by the stresses with additional sugars $[18,41]$. In only TSB, 112 (30\%) isolates produced biofilm, while adding $1 \%$ glucose, the number of biofilm positive isolates increased to $174(46.4 \%)$. This is consistent with the previous studies showing less positive results in TSB only medium $[18,42]$. Furthermore, the biofilm formation in BHI agar with $2 \%$ sucrose drastically increased number of biofilm producers to 229 (61\%). When the presence of icaAD genes was compared with TCP method, sensitivity increased on adding $1 \%$ glucose and $2 \%$ sucrose as compared to TSB only. These evidences suggest that biofilm formation by staphylococci depends on growth conditions. Indeed, the use of sugar as supplement in the media was found to be essential for biofilm formation [7, 18]. The use of additional sugar amount in a medium produces a stress condition that stimulates the fermentation reaction, resulting anaerobic condition that favors the production of PIA and consequently increasing biofilm production $[8,43]$. Taken together, these results indicate that the expression of ica gene is highly variable and induced by many factors including incorporation of sugar, salt, ethanol in the culture media $[6-8,18,40,44]$.
Detection of ica genes by PCR method has been demonstrated to be highly reliable to detect biofilm formation $[3,7,29]$. However, previous studies have shown evidences that presence of ica gene doesn't always correlate with biofilm production. For example; the study by deSilva demonstrated that only $59 \%$ of ica positive S. epidermidis isolates were found to be positive in CRA method [38]. In a study of Cafiso et al., 83.3\% of CRA and TCP positive isolates were ica positive [34]. We also observed the presence of icaAD genes in many biofilm-negative strains in phenotypic assays, indicating the importance of genotypic assay in in vitro biofilm detection. However, evidences showing ica independent biofilm production suggest that ica negative results may not always reveal the absence of biofilm production. For instance; the presence of accumulation associated protein $(a a p)$ or Bap homolog protein $(b h p)$ have been demonstrated to be responsible for biofilm production, suggesting the presence of PIA independent mechanisms in biofilm formation [37, 45-48].

\section{Conclusion}

The present study demonstrated the causal link between the presence of icaAD genes and biofilm production in the clinical staphylococcal isolates. Although TCP method was found to be superior to other phenotypic assays in terms of specificity and sensitivity, it was not well correlated with the genotypic assay. Taken together, these results suggest the use of genotypic assay along with the TM method in routine diagnostics to detect biofilm producers in clinical samples.

\section{Limitations}

Evaluation of biofilm production based merely on different nutrient supplements in vitro phenotypic assay may jeopardize the detections of biofilm production which depend on various factors. In addition, we examined presence of $i c a$ genes that are associated with PIA dependent biofilm production. This likely limits the detection of ica independent biofilm production.

\section{Additional files}

Additional file 1: Table S1. Frequency of Staphylococcal spp. in different clinical sample.

Additional file 2: Table S2. Correlation of Biofilm Production with ica genes.

Additional file 3: Table S3. Biofilm detection among S. aureus and CoNS by different methods.

Additional file 4: Table S4. Statistical evaluation of different phenotypic methods compared with standard TCP method. 


\begin{abstract}
Abbreviations
CoNS: Coagulase Negative Staphylococcus aureus; CRA: Congo-reg Agar; TM: tube method; TCP: tissue culture plate method; PCR: polymerase chain reaction; KIST: Kathmandu institute of science and technology; CVC: central venous catheter; ET: endotracheal tube; PIA: polysaccharide intercellular adhesion; PNAG: poly-N-acetylglucosamine; OD: optical density; ${ }^{\circ} \mathrm{C}$ : degree centigrade; icaADBC: intercellular adhesion operon containing icaA, icaD, icaB and icaC genes; aap: accumulation associated protein; bhp: Bap homolog protein
\end{abstract}

\section{Authors' contributions}

SM, primary author and corresponding author designed study methodology, performed laboratory investigation, prepared and revised the manuscript for submission. NRS and AS designed the study and edited manuscript. SP helped analyzing the result, proof reading and arranging references. AV helped in designing the study and proof reading of the manuscript. All authors read and approved the final manuscript.

\section{Author details \\ ${ }^{1}$ Tri-Chandra Multiple College, Tribhuvan University, Kathmandu, Nepal. ${ }^{2}$ Amity Institute of Microbial Technology, Amity University Uttar Pradesh, Noida, UP 201303, India. ${ }^{3}$ Central Department of Microbiology, Tribhuvan University, Kathmandu, Nepal. ${ }^{4}$ The University of Southern Mississippi, Hat- tiesburg, MS 39406, USA. ${ }^{5}$ Institute of Biotechnology, Zhejiang University, Hangzhou 310058, People's Republic of China.}

\section{Acknowledgements}

The authors are grateful to B \& B hospital, Gwarko and Kathmandu Institute of Science and technology (KIST) Medical College and teaching hospital, Imadol for allowing the collection of clinical samples.

\section{Competing interests}

The authors declare that they have no competing interests.

\section{Availability of data and materials}

All data obtained during this study are available within the article.

\section{Consent for publication}

Not applicable.

\section{Ethics approval and consent to participate}

Ethical approval (Ref No. 875) was obtained from Nepal Health Research Council, Kathmandu, Nepal before conducting the research. The study protocol was verified by Institutional Review Committee (IRC) of B \& B hospital, Gwarko and Kathmandu Institute of Science and Technology (KIST) Medical College and teaching hospital, Imadol where the samples were collected from the patients for the standard care. Informed written consent to use the samples was obtained from each patient in this study.

\section{Funding}

No specific funding for this study was received.

\section{Publisher's Note}

Springer Nature remains neutral with regard to jurisdictional claims in published maps and institutional affiliations.

Received: 2 August 2018 Accepted: 3 October 2018

Published online: 10 October 2018

\section{References}

1. Donlan RM, Costerton JW. Biofilms: survival mechanisms of clinically relevant microorganisms. Clin Microbiol Rev. 2002;15(2):167-93.

2. Gotz F. Staphylococcus and biofilms. Mol Microbiol. 2002;43(6):1367-78.

3. O'Gara JP. ica and beyond: biofilm mechanisms and regulation in Staphylococcus epidermidis and Staphylococcus aureus. FEMS Microbiol Lett. 2007;270(2):179-88.

4. Cerca N, Martins S, Cerca F, Jefferson KK, Pier GB, Oliveira R, Azeredo J. Comparative assessment of antibiotic susceptibility of coagulase-negative staphylococci in biofilm versus planktonic culture as assessed by bacterial enumeration or rapid XTT colorimetry. J Antimicrob Chemother. 2005;56(2):331-6.

5. Bazzoun DA, Harastani HH, Shehabi AA, Tokajian ST. Molecular typing of Staphylococcus aureus collected from a Major Hospital in Amman, Jordan. J Infect Dev Ctries. 2014;8(4):441-7.

6. Mack D, Fischer W, Krokotsch A, Leopold K, Hartmann R, Egge H, Laufs R. The intercellular adhesin involved in biofilm accumulation of Staphylococcus epidermidis is a linear beta-1,6-linked glucosaminoglycan: purification and structural analysis. J Bacteriol. 1996;178(1):175-83.

7. Oliveira A, Cunha Mde L. Comparison of methods for the detection of biofilm production in coagulase-negative staphylococci. BMC Res Notes. 2010;3:260.

8. Vuong C, Kocianova S, Voyich JM, Yao Y, Fischer ER, DeLeo FR, Otto M. A crucial role for exopolysaccharide modification in bacterial biofilm formation, immune evasion, and virulence. J Biol Chem. 2004;279(52):54881-6.

9. Wojtyczka RDOK, Kępa M, Idzik D, Dziedzic A, MularzT, Krawczyk M, Miklasińska M, Wąsik TJ. Biofilm formation and antimicrobial susceptibility of Staphylococcus epidermidis strains from a hospital environment. Int J Environ Res Public Health. 2014;11(5):4619-33.

10. Dobnisky SK, Rohde H, Bartscht K, Knobloch J, Horskotte M, Dietrich M. Glucose related dissociation between ica ADBC transcription and biofilm expression by Staphylococcus epidermidis: evidence for an additional factor required for polysaccharide intercellular adhesion synthesis. J Bacteriol. 2003;185:2879-86.

11. Tang Y-W. SCW: Staphylococcus aureus: an old pathogen with new weapons. Clin Lab Med. 2010;30:179-208.

12. Stewart PS, Costerton JW. Antibiotic resistance of bacteria in biofilms. Lancet. 2001;358(9276):135-8.

13. Hamilton MA. Testing antimicrobials against biofilm bacteria. J AOAC Int. 2002;85(2):479-85.

14. von Eiff CPG, Heilmann C. Pathogenesis of infections due to coagulasenegative staphylococci. Lancet Infect Dis. 2002;2(11):677-85.

15. Klingenberg CAE, Rønnestad A, Sollid JE, Abrahamsen TG, Kjeldsen G, Flaegstad T. Coagulase-negative staphylococcal sepsis in neonates. Association between antibiotic resistance, biofilm formation and the host inflammatory response. Pediatr Infect Dis J. 2005;24(9):817-22.

16. Arciola CR, Campoccia D, Ravaioli S, Montanaro L. Polysaccharide intercellular adhesin in biofilm: structural and regulatory aspects. Front Cell Infect Microbiol. 2015;5:7.

17. Nathan K, Archer M, Mazaitis J, Costerton J, Powers M, Mark E. Staphylococcus aureus biofilms. Virulence. 2011;2(5):445-59.

18. MathurTSS, Khan S, Upadhyay DJ, Fatma T, Rattan A. Detection of biofilm formation among the clinical isolates of staphylococci: An evaluation of three different screening methods. Indian J Med Microbiol. 2006;24:25-9.

19. Cheeseburg M. District laboratory practice in tropical countries. Cambridge: Cambridge University Press; 2006.

20. Freeman DJ, Falkiner FR, Keane CT. New method for detecting slime production by coagulase-negative staphylococci. J Clin Pathol. 1989;42(8):872-4.

21. Christensen GD, Simpson WA, Younger JJ, Baddour LM, Barrett FF, Melton DM, Beachey EH. Adherence of coagulase-negative staphylococci to plastic tissue culture plates: a quantitative model for the adherence of staphylococci to medical devices. J Clin Microbiol. 1985;22(6):996-1006.

22. Cramton SE, Gerke C, Schnell NF, Nichols WW, Gotz F. The intercellular adhesion (ica) locus is present in Staphylococcus aureus and is required for biofilm formation. Infect Immun. 1999;67(10):5427-33.

23. Atshan SS, Nor Shamsudin M, Sekawi Z, Lung LT, Hamat RA, Karunanidhi A, Mateg Ali A, Ghaznavi-Rad E, Ghasemzadeh-Moghaddam H, Chong Seng JS, et al. Prevalence of adhesion and regulation of biofilm-related genes in different clones of Staphylococcus aureus. J Biomed Biotechnol. 2012;2012:976972.

24. Shrestha LB, Bhattarai NR, Khanal B. Antibiotic resistance and biofilm formation among coagulase-negative staphylococci isolated from clinical samples at a tertiary care hospital of eastern Nepal. Antimicrob Resist infect Control. 2017;6:89.

25. Molnar C, Hevessy Z, Rozgonyi F, Gemmell CG. Pathogenicity and virulence of coagulase negative staphylococci in relation to adherence, hydrophobicity, and toxin production in vitro. J Clin Pathol. 1994;47(8):743-8.

26. Otto M. Staphylococcus epidermidis-the 'accidental' pathogen. Nat Rev Microbiol. 2009:7(8):555-67. 
27. Widerstrom M, Wistrom J, Sjostedt A, Monsen T. Coagulase-negative staphylococci: update on the molecular epidemiology and clinical presentation, with a focus on Staphylococcus epidermidis and Staphylococcus saprophyticus. Eur J Clin Microbiol Infect Dis. 2012;31(1):7-20.

28. Costerton JW, Stewart PS, Greenberg EP. Bacterial biofilms: a common cause of persistent infections. Science. 1999;284(5418):1318-22.

29. Arciola $C R$, Baldassarri L, Montanaro L. Presence of icaA and icaD genes and slime production in a collection of staphylococcal strains from catheter-associated infections. J Clin Microbiol. 2001;39(6):2151-6.

30. Arciola CR, Collamati S, Donati E, Montanaro L. A rapid PCR method for the detection of slime-producing strains of Staphylococcus epidermidis and S. aureus in periprosthesis infections. Diagn Mol Pathol. 2001;10(2):130-7.

31. Nasr RA. Biofilm formation and presence of icaAD gene in clinical isolates of staphylococci. Egypt J Med Human Genet. 2012;13(3):6.

32. Namvar AE, Asghari B, Ezzatifar F, Azizi G, Lari AR. Detection of the intercellular adhesion gene cluster (ica) in clinical Staphylococcus aureus isolates. GMS Hyg Infect Control. 2013;8(1):Doc03.

33. Cue D. Genetic regulation of the intercellular adhesion locus in staphylococci. Front Cell Infect Microbiol. 2012;2:38.

34. Cafiso V, Bertuccio T, Santagati M, Campanile F, Amicosante G, Perilli MG, Selan L, Artini M, Nicoletti G, Stefani S. Presence of the ica operon in clinical isolates of Staphylococcus epidermidis and its role in biofilm production. Clin Microbiol Infect. 2004;10(12):1081-8.

35. Gad GF, El-Feky MA, El-Rehewy MS, Hassan MA, Abolella H, El-Baky RM. Detection of icaA, icaD genes and biofilm production by Staphylococcus aureus and Staphylococcus epidermidis isolated from urinary tract catheterized patients. J Infect Dev Ctries. 2009;3(5):342-51.

36. Ruzicka F, Hola V, Votava M, Tejkalova R, Horvat R, Heroldova M, Woznicova V. Biofilm detection and the clinical significance of Staphylococcus epidermidis isolates. Folia Microbiol. 2004;49(5):596-600.

37. Los R, Sawicki R, Juda M, Stankevic M, Rybojad P, Sawicki M, Malm A, Ginalska G. A comparative analysis of phenotypic and genotypic methods for the determination of the biofilm-forming abilities of Staphylococcus epidermidis. FEMS Microbiol Lett. 2010;310(2):97-103.

38. de Silva GD, Kantzanou M, Justice A, Massey RC, Wilkinson AR, Day NP, Peacock SJ. The ica operon and biofilm production in coagulase-negative Staphylococci associated with carriage and disease in a neonatal intensive care unit. J Clin Microbiol. 2002;40(2):382-8.
39. Arciola CR, Campoccia D, Gamberini S, Cervellati M, Donati E, Montanaro L. Detection of slime production by means of an optimised Congo red agar plate test based on a colourimetric scale in Staphylococcus epidermidis clinical isolates genotyped for ica locus. Biomaterials. 2002;23(21):4233-9.

40. Fitzpatrick F, Humphreys H, O'Gara JP. The genetics of staphylococcal biofilm formation-will a greater understanding of pathogenesis lead to better management of device-related infection? Clin Microbiol Infect. 2005;11(12):967-73.

41. Cho SH, Naber K, Hacker J, Ziebuhr W. Detection of the icaADBC gene cluster and biofilm formation in Staphylococcus epidermidis isolates from catheter-related urinary tract infections. Int J Antimicrob Agents. 2002;19(6):570-5.

42. Johannes KM. Evaluation of different methods of biofilm formation in Staphylococcus aureus. Med Microbiol Immunol. 2002;191:7.

43. Arciola CR, Campoccia D, Baldassarri L, Donati ME, Pirini V, Gamberini S, Montanaro L. Detection of biofilm formation in Staphylococcus epidermidis from implant infections Comparison of a PCR-method that recognizes the presence of ica genes with two classic phenotypic methods. J Biomed Mater Res. 2006;76(2):425-30.

44. Mack D, Rohde H, Dobinsky S, Riedewald J, Nedelmann M, Knobloch $\mathrm{JK}$, Elsner HA, Feucht HH. Identification of three essential regulatory gene loci governing expression of Staphylococcus epidermidis polysaccharide intercellular adhesin and biofilm formation. Infect Immun. 2000;68(7):3799-807.

45. Rohde H, Burandt EC, Siemssen N, Frommelt L, Burdelski C, Wurster S, Scherpe S, Davies AP, Harris LG, Horstkotte MA, et al. Polysaccharide intercellular adhesin or protein factors in biofilm accumulation of Staphylococcus epidermidis and Staphylococcus aureus isolated from prosthetic hip and knee joint infections. Biomaterials. 2007;28(9):1711-20.

46. McCann MT, Gilmore BF, Gorman SP. Staphylococcus epidermidis devicerelated infections: pathogenesis and clinical management. J Pharm Pharmacol. 2008:60(12):1551-71.

47. Qin Z, Yang X, Yang L, Jiang J, Ou Y, Molin S, Qu D. Formation and properties of in vitro biofilms of ica-negative Staphylococcus epidermidis clinical isolates. J Med Microbiol. 2007;56(Pt 1):83-93.

48. Jiang J. Influence of ica transcription on biofilm phenotype of Staphylococcus epidermidis clinical isolates. Shanghai Med J. 2006;29:5.
Ready to submit your research? Choose BMC and benefit from:

- fast, convenient online submission

- thorough peer review by experienced researchers in your field

- rapid publication on acceptance

- support for research data, including large and complex data types

- gold Open Access which fosters wider collaboration and increased citations

- maximum visibility for your research: over $100 \mathrm{M}$ website views per year

At BMC, research is always in progress.

Learn more biomedcentral.com/submissions 